\title{
Protein crystallization assisted by the crystallophore.
}

\author{
E. Girard ${ }^{1}$, Z. Alsalman ${ }^{1}$, A. Robin ${ }^{1}$, S. Engilberge ${ }^{1}$, A. Roux ${ }^{2}$, F. Riobé ${ }^{2}$, O. Maury ${ }^{2}$ \\ ${ }^{1}$ Univ. Grenoble Alpes, CEA, CNRS, IBS, F-38000 Grenoble, France, \\ ${ }^{2}$ Univ. Lyon, ENS de Lyon, CNRS UMR 5182, Université Claude Bernard Lyon 1, Laboratoire de Chimie, F-69342 Lyon, France.
}

$$
\text { eric.girard@ibs.fr }
$$

Obtaining crystals and solving the phase problem remain major hurdles encountered by bio-crystallographers in their race to get new high-quality structures. The crystallophore, Xo4, is a family of nucleating and phasing molecules based on lanthanide complexes. TbXo4 was the first molecule of this family to be described [1].

Results obtained on more than fifteen proteins will be described and will show that Tb-Xo4 is an efficient tool to promote protein crystallization. Among these results, we will show that (i) Tb-Xo4 increases the number of crystallization conditions by promoting unique ones [1,2] (ii) the crystalline forms promoted by the crystallophore bypass crystal defects often encountered by crystallographers such as low-resolution diffracting samples or crystals with twinning [3] and (iii) the crystallization reproducibility is largely improved, a particular issue in structure-based drug design.

Contrary to the dogma that crystallization can only be promoted from pure protein sample, we have shown that crystals can be obtained from enriched fractions containing several proteins [3] leading to the structure determination of a protein complex [4]. Even more unexpected, the crystallophore is able to induce crystallization directly from the protein solution, as exemplified by the crystallization of hen egg white lysozyme in water [5].

Finally, we will also present preliminary results on several crystallophore variants showing complementarity with $\mathrm{Tb}$-Xo4 thus enlarging the success in defining exploitable crystallization conditions.

Altogether, crystallophore is an efficient solution for protein crystallization and structure determination in the bio-crystallographer toolbox.

[1] Engilberge, S., Riobé, F., Di Pietro, S., Lassalle, L., Coquelle, N., Arnaud, C.-A., Pitrat, D., Mulatier, J.-C., Madern, D., Breyton, C., Maury, O. \& Girard, E. (2017). Chem. Sci. 8, 5909-5917.

[2] Jiang, T., Roux, A., Engilberge, S., Alsalman, Z., Di Pietro, S., Franzetti, B., Riobé, F., Maury, O. \& Girard, E. (2020). Crystal Growth \& Design. $20,5322-5329$.

[3] Engilberge, S., Wagner, T., Santoni, G., Breyton, C., Shima, S., Franzetti, B., Riobé, F., Maury, O. \& Girard, E. (2019). Journal of Applied Crystallography. 52, 722-731.

[4] Vögeli, B., Engilberge, S., Girard, E., Riobé, F., Maury, O., Erb, T. J., Shima, S. \& Wagner, T. (2018). Proceedings of the National Academy of Sciences. 115, 3380-3385.

[5] de Wijn, R., Rollet, K., Engilberge, S., McEwen, A. G., Hennig, O., Betat, H., Mörl, M., Riobé, F., Maury, O., Girard, E., Bénas, P., Lorber, B. \& Sauter, C. (2020). Crystals. 10, 65.

Keywords: Crystallisation; crystallophore; nucleating agents; structure determination; phasing.

Authors acknowledge financial supports from the Fondation Maison de la Chimie, Agence Nationale de la Recherche (ANR Ln23-13BS07-0007-01) and Region AuRA for (program Xo4-2.0). 